Check for updates

Cite this: RSC Adv., 2017, 7, 39583

\title{
A computational approach towards understanding hydrogen gas adsorption in Co-MIL-88A $\uparrow$
}

\begin{abstract}
Nguyen Thi Xuan Huynh, ${ }^{\text {ab }} \mathrm{O}$ My Na, (D) a Viorel Chihaia (D) ${ }^{c}$ and Do Ngoc Son (D) *a
Unsaturated metal centers in metal-organic framework MIL-88A are able to significantly enhance the amount of gas adsorbed at ambient temperatures and low pressures. This material has been investigated for various applications; however, it has not yet been tested for hydrogen storage. In this research, we examined the interaction of hydrogen gas $\left(\mathrm{H}_{2}\right)$ with Co-MIL-88A by using the van der Waals dispersioncorrected density functional theory calculations. The $\mathrm{H}_{2}$ molecule was found to adsorb most favorably at the hollow site of the metal trimers in Co-MIL-88A because of the maximum overlap between the bonding state of the $\mathrm{H}_{2}$ molecule and the total density of state of the Co-MIL-88A. In addition, the hydrogen adsorption isotherms were also assessed by grand canonical Monte Carlo simulations. The results showed that $\mathrm{CO}-\mathrm{MIL}-88 \mathrm{~A}$ is one of the most effective $\mathrm{H}_{2}$ storage materials.
\end{abstract}

Received 23rd May 2017

Accepted 7th August 2017

DOI: 10.1039/c7ra05801b

rsc.li/rsc-advances
Cobalt, a transitional metal, plays an important role in improving the $\mathrm{H}_{2}$ uptake capacity of MOFs. For example, $\mathrm{Co}_{2}$ (dobpdc) showed the highest gravimetric $\mathrm{H}_{2}$ uptake among $\mathrm{M}_{2}$ (dobpdc), where $\mathrm{M}$ is $\mathrm{Mg}, \mathrm{Mn}, \mathrm{Fe}, \mathrm{Co}$, Ni or $\mathrm{Zn} .{ }^{20}$ The result of Zhou and co-workers pointed out that the higher the temperature, the larger the uptake of $\mathrm{H}_{2}$ adsorbed in Co-MOF-74 [also called $\mathrm{CPO}-27-\mathrm{Co}, \mathrm{Co}_{2}(\mathrm{dobdc})$ or $\mathrm{Co}_{2}(\mathrm{dhtp}){ }^{21}{ }^{21}$ Their result showed, at $150 \mathrm{~K}$, Co-MOF-74 has the highest $\mathrm{H}_{2}$ uptake in comparison to M-MOF-74 (M = Mg, Mn, Ni, Zn) (see the ESI of ref. 21). Therefore, we expect that the Co-based MIL-88A (hereafter called Co-MIL-88A) with the coordinatively unsaturated Co metal sites (CUS) ${ }^{22}$ also offer a high hydrogen sorption capacity, especially at ambient temperatures.

In this work, we evaluate the hydrogen adsorption capability of the Co-MIL-88A and explain the physical origin for the interaction between $\mathrm{H}_{2}$ molecule and the Co-MIL-88A MOF. We first search for the most favorable adsorption sites of $\mathrm{H}_{2}$ via computing the adsorption energy and then analyze electronic properties based on the van der Waals dispersion-corrected density functional theory calculations. We then calculate hydrogen adsorption isotherms of the Co-MIL-88A by using grand canonical Monte Carlo (GCMC) simulations.

\section{Computational methods}

For the study of favorable adsorption sites and electronic structure properties, we employed the Vienna ab initio simulation package (VASP) ${ }^{23}$ for the van der Waals dispersioncorrected (vdW-DF version) density functional theory (DFT) calculations. The plane-wave basis set with the cut-off energy of $700 \mathrm{eV}$, the revised Perdew-Burke-Ernzerhof (revBPE) functional for the exchange-correlation energy, ${ }^{\mathbf{2 4 2 5}}$ and the projector-augmented-wave method for the electron-ion 
interaction $^{\mathbf{2 6 , 2 7}}$ were used to perform the calculations. The surface Brillouin-zone integrations were performed by using the Monkhorst and Pack $k$-point sampling technique ${ }^{28}$ with the $4 \times$ $4 \times 4$ mesh grid and the gamma point at the center. The Methfessel-Paxton smearing ${ }^{29}$ of order 1 was used for the geometry relaxation with the smearing width of $0.1 \mathrm{eV}$. However, the linear tetrahedron method with Blöchl corrections ${ }^{30}$ was employed for the calculations of total energy.

The electronic structure properties were elucidated through the analysis of the density derived electrostatic and chemical (DDEC) net atomic charge, ${ }^{31}$ the Bader point charge, ${ }^{32}$ the electronic density of state (DOS), and the charge density difference (CDD).

For estimating the binding strength of $\mathrm{H}_{2}$ with Co-MIL-88A, we calculated the adsorption energy $E_{\text {ads }}$ of $\mathrm{H}_{2}$ by using the following formula:

$$
E_{\mathrm{ads}}=-E_{\mathrm{b}}=E_{\mathrm{MOF}+\mathrm{H}_{2}}-\left(E_{\mathrm{MOF}}+E_{\mathrm{H}_{2}}\right) .
$$

here, $E_{\mathrm{MOF}+\mathrm{H}_{2}}, E_{\mathrm{MOF}}, E_{\mathrm{H}_{2}}$ is the total energy of the Co-MIL-88A + $\mathrm{H}_{2}$ system, the isolated Co-MIL-88A, and the isolated $\mathrm{H}_{2}$ molecule, respectively. The binding energy of the $\mathrm{H}_{2}$ molecule and the Co-MIL-88A is denoted by $E_{\mathrm{b}}$.

Grand canonical Monte Carlo (GCMC) simulations were used to compute the gravimetric loadings of hydrogen gas in the Co-MIL-88A MOF by using the simulation package RASPA, which is a molecular simulation software for nanoporous materials. $^{33}$ These simulations were performed in the $V T \mu$ (constant volume, temperature, and chemical potential) ensembles at two different temperatures ( $77 \mathrm{~K}$ and $298 \mathrm{~K}$ ) for pressures up to 100 bar. The number of MC steps was $3 \times 10^{5}$ for the random insertion, deletion, translation, and rotation of $\mathrm{H}_{2}$ molecules in the simulation box of the Co-MIL-88A framework of $3 \times 3 \times 2$ times of the unit cell. The framework was kept rigid during the simulation process, while hydrogen molecule freely moves in the MOF structure. The interaction between the $\mathrm{H}_{2}$ gas and the atoms (C, O, H and Co) of the MOF were described through the Lennard-Jones (LJ) 6-12 potential (the first two terms, in square parenthesis) and the electrostatic potential (the last term), presented by

$$
U\left(r_{i j}\right)=4 \varepsilon_{i j}\left[\left(\frac{\sigma_{i j}}{r_{i j}}\right)^{12}-\left(\frac{\sigma_{i j}}{r_{i j}}\right)^{6}\right]+\frac{1}{4 \pi \varepsilon_{0}} \frac{q_{i} q_{j}}{r_{i j}},
$$

where $U$ is the potential energy between a pair of atoms $i$ and $j$ at a distance $r_{i j}$; $\varepsilon_{0}$ is the dielectric constant; $q_{i}$ is the partial charge of atom $i$ obtained from the DDEC atomic net charge calculation based on the DFT method. The parameters $\varepsilon_{i j}$ and $\sigma_{i j}$ are the LJ potential well depth and diameter, respectively. These parameters were calculated by using the Lorentz-Berthelot mixing rule:

$$
\varepsilon_{i j}=\sqrt{\varepsilon_{i} \varepsilon_{j}}, \quad \sigma_{i j}=\frac{1}{2}\left(\sigma_{i}+\sigma_{j}\right) .
$$

here, the $\mathrm{LJ}$ parameters for atom $i\left(\sigma_{i}, \varepsilon_{i}\right)$ were taken from generic force fields for MOFs in RASPA software package, ${ }^{33}$ listed in Table 1.
Table 1 The LJ parameters for the atom types and the partial charges used in our GCMC simulations

\begin{tabular}{lrrl}
\hline Atom type & $\varepsilon / k_{\mathrm{B}}(\mathrm{K})$ & $\sigma(\AA)$ & DDEC charge $\left(\mathrm{e}^{-}\right)$ \\
\hline $\mathrm{Co}$ & 7.05 & 2.56 & 1.158 \\
$\mathrm{C}$ (on the metal trimer) & 47.86 & 3.47 & 0.734 \\
$\mathrm{C}$ (on the linker) & 47.86 & 3.47 & -0.169 \\
$\mathrm{O}$ & 48.16 & 3.03 & -0.559 \\
$\mu_{3}-\mathrm{O}$ & 48.16 & 3.03 & -0.858 \\
$\mathrm{H}$ & 7.65 & 2.85 & 0.117 \\
$\mathrm{H}_{\text {com }}$ of $\mathrm{H}_{2}$ molecule & 36.70 & 2.96 & -0.936 \\
\hline
\end{tabular}

The Lennard-Jones interaction is neglected beyond the unique cutoff radius of $12 \AA$. The electrostatic equation was calculated by the Ewald summation technique. For the hydrogen molecule, a single LJ interaction site model at the center of mass $\left(\mathrm{H}_{\text {com }}\right)$ was used with the LJ parameters taken from the TraPPE force field. These parameters are $\sigma_{\mathrm{H}_{\text {com }}}=2.96 \AA$ and $\varepsilon_{\mathrm{H}_{\mathrm{com}}} / k_{\mathrm{B}}=36.70 \mathrm{~K}$ with the $\mathrm{H}-\mathrm{H}$ bond length of $0.74 \AA$.

\section{Results and discussion}

\subsection{Geometry optimization of Co-MIL-88A}

In this work, the Co-MIL-88A was designed with the chemical formula $\left[\left\{\mathrm{Co}_{3} \mathrm{O}\left(-\mathrm{O}_{2} \mathrm{C}-\mathrm{C}_{2} \mathrm{H}_{2}-\mathrm{CO}_{2}-\right)_{3}\right\}\right]_{n}$ having a threedimensional hexagonal structure consisting of the trimers of Co octahedra linked to the fumarate ligands, where $n$ is the number of chemical formula units. This structure has the neutral charge with the Co atoms in a closed shell configuration due to bonding with the other atoms of the MOF. Fig. 1 shows the structure of the unit cell of Co-MIL-88A with $n=2$. The dimensions of the unit cell are $a, b$, and $c$, where $a=b$ and the angles $\alpha=\beta=90^{\circ}, \gamma=120^{\circ}$.

After the primary unit cell for Co-MIL-88A was designed, the geometry optimization for Co-MIL-88A was performed for its volume and ionic positions. The ionic positions were relaxed by using the van der Waals-density functional (vdW-DF) of Langreth and Lundqvist et al. ${ }^{34}$

To obtain the optimized volume of the unit cell, we calculated the total energy of the unit cell for the following conditions. For several fixed values of $c / a$ ratio between 1.25 and 1.39, we varied the $a$ lattice constant from 10.58 to $11.98 \AA$ in $0.2 \AA$ grids. We then fitted the unit cell volume to the Murnaghan's equation of state ${ }^{35}$ based on the obtained total energy versus the structural parameter $a$ and $c / a$. Fig. 2a shows that the minimum energy is found at different $a$ for the different $c / a$. The collection of the minimum energy points as a function of the lattice constant $a$ and the $c / a$ ratio is presented in Fig. $2 \mathrm{~b}$ and $\mathrm{c}$, respectively. The detailed values of the minimum energies are listed in Table 2 . On the basis of these curves, we found an optimized lattice constant of $a=b=11.222 \AA$ and $c=14.719 \AA$, corresponding to a volume of $1605.34 \AA^{3}$. This volume is close to the experimental value of $1589 \AA^{3}$ for the Fe-MIL-88A. ${ }^{36}$ It should be noted that the ionic radius of $\mathrm{Co}^{3+}$ is $0.69 \AA$ which is the same as that of the $\mathrm{Fe}^{3+}$ ion. 

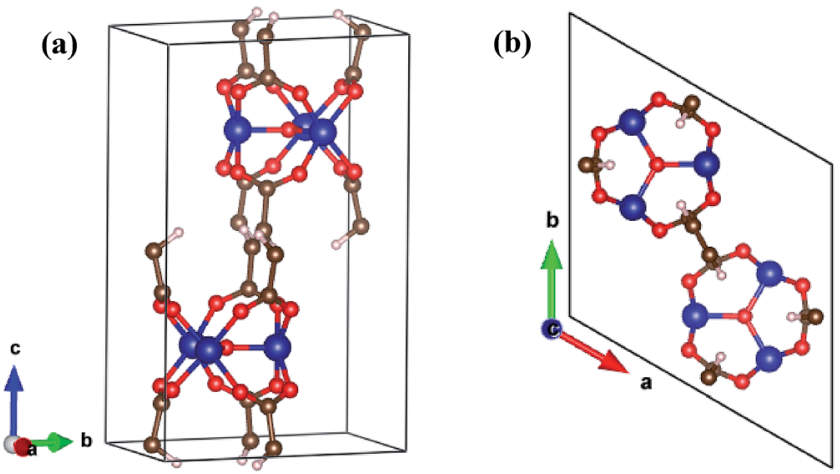

(c)

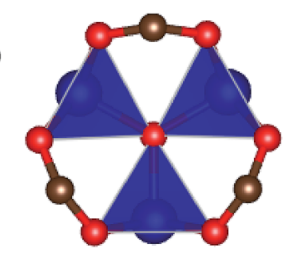

(d)

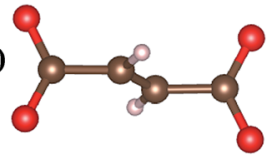

Fig. 1 The structure of Co-MIL-88A: side view (a) and top view (b) of the unit cell, the $\mu_{3}$-O-centered trimer of Co metals (c), and the fumarate linker of the MOF (d). The blue, red, brown, and white colored balls represent the cobalt, oxygen, carbon, and hydrogen atoms, respectively.
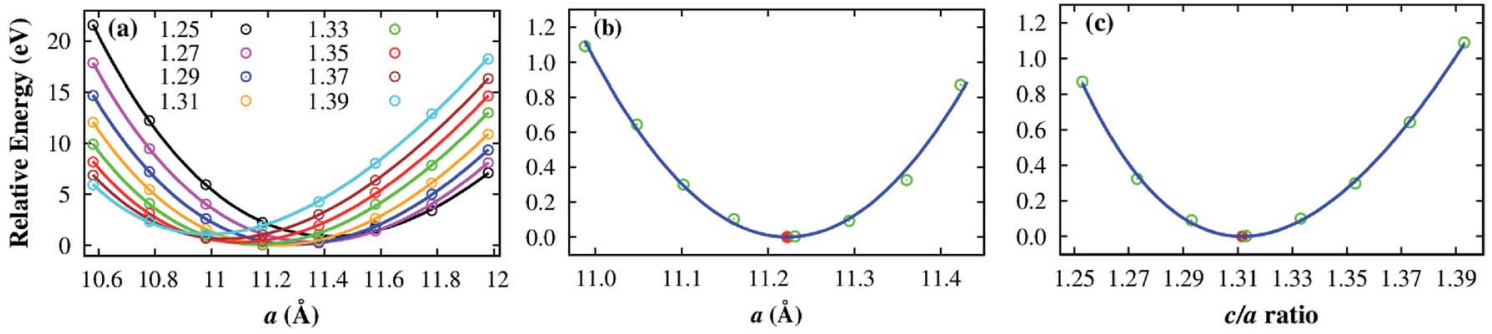

Fig. 2 Relative energy as a function of the lattice constant a for each c/a ratio (a). The solid lines are the fitting curves, while the points are the calculated values. The minimum energy points are plotted versus the lattice constant $a$ (b) and the c/a ratio (c).

Table 2 The minimum energy of the Co-MIL-88A unit cell and the corresponding structural parameters

\begin{tabular}{lll}
\hline$c / a$ ratio & Lattice constant $a(\AA)$ & Minimum total energy $(\mathrm{eV})$ \\
\hline 1.253 & 11.42 & -358.01 \\
1.273 & 11.36 & -358.55 \\
1.293 & 11.29 & -358.78 \\
1.313 & 11.23 & -358.87 \\
1.333 & 11.16 & -358.77 \\
1.353 & 11.10 & -358.58 \\
1.373 & 11.05 & -358.23 \\
1.393 & 10.99 & -357.79 \\
\hline
\end{tabular}

\subsection{Favorable hydrogen adsorption sites and electronic properties}

After obtaining the Co-MIL-88A unit cell with the optimized lattice constants, a hydrogen molecule $\left(\mathrm{H}_{2}\right)$ is loaded into the unit cell at many different sites. We then performed geometry optimization and calculated the adsorption energy of $\mathrm{H}_{2}$. The results are listed in Table 3 together with the average bond length of $\mathrm{H}_{2}$ to the closest atoms of the MOF. From these calculations, we found the favorable adsorption sites of $\mathrm{H}_{2}$. Fig. $3 a$ and $b$ are the adsorption configurations of $\mathrm{H}_{2}$ on the hollow and ligand sites, and Fig. $3 \mathrm{c}$ and $\mathrm{d}$ are the side-on and end-on configurations of $\mathrm{H}_{2}$ over the Co metal atom. For the metal end-on configuration, the $\mathrm{H}$ atoms of $\mathrm{H}_{2}$ molecule and the nearest Co atom form an angle of $180^{\circ}$. The hollow site is formed by four oxygen atoms $(\mathrm{O} 1, \mathrm{O} 2, \mathrm{O} 3$ and $\mathrm{O} 4)$ of the MOF. The hydrogen molecule adsorbs in a parallel configuration with the plane of these oxygen atoms. For the ligand site, $\mathrm{H}_{2}$ adsorbs over the fumarate with the $\mathrm{H}-\mathrm{H}$ bond perpendicular to the line connecting $\mathrm{C} 1$ and $\mathrm{C} 2$. The average distance of $\mathrm{H}_{2}$ to the reference atoms of the MOF $(3.20 \AA)$ for the most favorable adsorption site (i.e. the hollow site) is comparable to that for the side-on and end-on ones on the metal site, $c a$. $3.15 \AA$, but significantly shorter than that for the binding to the ligand, $3.41 \AA$.

From Table 3, we see that, on the basis of the adsorption energy, the favorable adsorption site is in the order of hollow > ligand > metal side-on > metal end-on. The most favorable adsorption of $\mathrm{H}_{2}$ is at the hollow site with a binding energy of $13.72 \mathrm{~kJ} \mathrm{~mol}^{-1}$. Although, hydrogen molecule was also found to have strong adsorption at the hollow site of MOF-5, ${ }^{37-39}$ it is usually expected that the strong hydrogen bonding is at the metal site. Surprisingly, the adsorption is even less favorable on the metal site than on the organic ligand, which is different with common expectation. For instance, several authors found that the hydrogen binding energies on the ligand were smaller than those on the metal. ${ }^{40,41}$ The reason for this strong adsorption of $\mathrm{H}_{2}$ on the organic ligand of the Co-MIL-88A may be due to the relatively short fumarate bridge. Thereby, the metal and oxygen atoms may interact with the $\mathrm{H}_{2}$ located at the hollow and ligand sites. This point will be discussed through the analysis of the electronic density of state in the below part. For the $\mathrm{H}_{2}$ adsorbed end-on configuration on the metal site, the binding energy of 
Table 3 The adsorption sites, adsorption energy, structural parameters, and electronic property of $\mathrm{H}_{2}$ in Co-MIL-88A. The average distance between the $\mathrm{H}_{2}$ molecule and the reference atoms of the MOF is denoted by $d_{\mathrm{H}_{2}-\mathrm{A}}$ and the Bader point charge of $\mathrm{H}_{2}$ is denoted by $q_{\mathrm{H}_{2}}$

\begin{tabular}{lllll}
\hline Sites & $E_{\text {ads }}\left(\mathrm{kJ} \mathrm{mol}^{-1}\right)$ & $E_{\mathrm{b}}\left(\mathrm{kJ} \mathrm{mol}^{-1}\right)$ & $d_{\mathrm{H}_{2}-\mathrm{A}}(\AA)$ & $q_{\mathrm{H}_{2}}(\mathrm{e})$ \\
\hline Hollow & -13.72 & 13.72 & 3.20 & -0.0002 \\
Ligand & -10.76 & 10.76 & 3.41 & -0.0006 \\
Metal (side-on) & -10.61 & 10.61 & 3.14 & -0.0038 \\
Metal (end-on) & -6.50 & 6.50 & 3.15 & -0.0006
\end{tabular}

this configuration is the smallest compared with that of the others.

All the vibrational modes for the $\mathrm{H}_{2}$ molecule showed positive values implying that the hollow, ligand, metal end-on and metal side-on configurations are local minima of the $\mathrm{H}_{2}$ adsorption (see Table $\mathrm{S} 1$ in ESI $\dagger$ ). We also computed the $\mathrm{H}-\mathrm{H}$ stretching frequency shift $(\Delta \nu)$ for the different adsorption sites relative to the stretching frequency of the free $\mathrm{H}_{2}$ molecule. Table 4 shows that the stretching frequency shift is about $27-46 \mathrm{~cm}^{-1}$, which is much smaller than those in the work of Solans-Monfort. ${ }^{42}$ This no significant perturbation of the
Table 4 The $\mathrm{H}-\mathrm{H}$ stretching frequency shift $(\Delta \nu)$ and the zero-point energy for the adsorbed $\mathrm{H}_{2}$ at the favorable adsorption sites

\begin{tabular}{lll}
\hline Sites & $\Delta \nu^{a}\left(\mathrm{~cm}^{-1}\right)$ & Zero-point energy $\left(\mathrm{kJ} \mathrm{mol}^{-1}\right)$ \\
\hline Hollow & -46 & 31.95 \\
Ligand & -46 & 31.76 \\
Metal side-on & -39 & 30.12 \\
Metal end-on & -27 & 30.08
\end{tabular}

${ }^{a}$ The $\mathrm{H}-\mathrm{H}$ harmonic stretching frequency shift with respect to the free $\mathrm{H}_{2}$ stretching frequency, $\Delta \nu=\nu\left(\mathrm{MOF}+\mathrm{H}_{2}\right)-\nu\left(\right.$ isolated $\left.\mathrm{H}_{2}\right)$.

stretching frequency suggests that the charge transfer between the MOF and the adsorbed $\mathrm{H}_{2}$ should be little. Additionally, the zero-point vibration energy is about $1.8 \mathrm{~kJ} \mathrm{~mol}^{-1}$ larger for the hollow site compared to the metal sites, but it is still smaller than the electronic energy difference of about $3 \mathrm{~kJ} \mathrm{~mol}^{-1}$. Thereby, the hollow site is the likely binding site even including zero-point energy corrections.

Although the binding energy of $\mathrm{H}_{2}$ with the Co-MIL-88A is much smaller than DOE's target binding energy of $20-25 \mathrm{~kJ} \mathrm{~mol}^{-1,43,44}$ it is greater than that of many MOFs

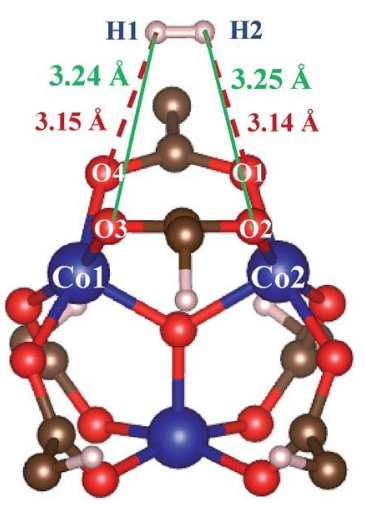

a) Hollow

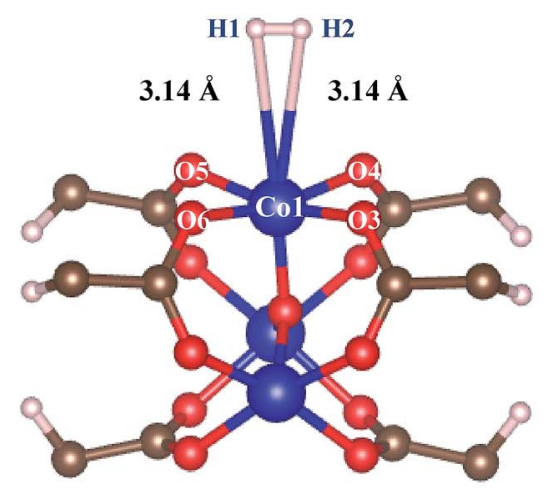

c) Metal (side-on)

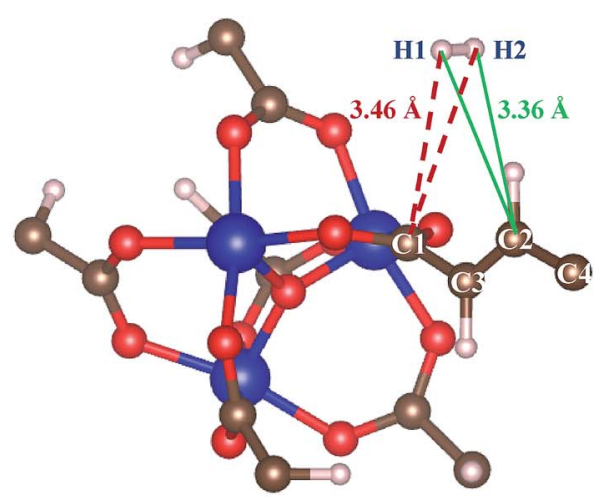

b) Ligand

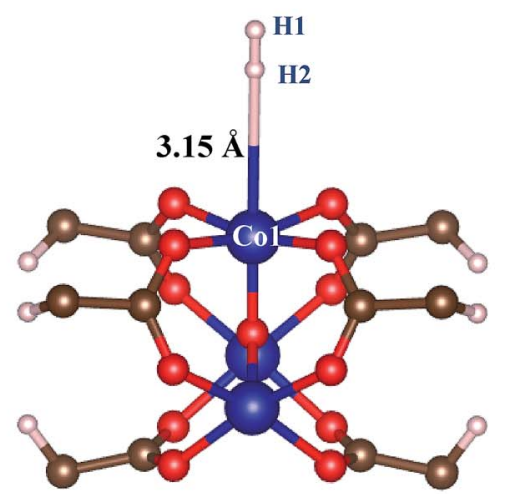

d) Metal (end-on)

Fig. 3 The favorable adsorption configurations of $\mathrm{H}_{2}$ in $\mathrm{Co}-\mathrm{MIL}-88 \mathrm{~A}$. The bond distance to the reference atoms is correspondingly shown for each configuration. 
currently achieved $\sim 7-10 \mathrm{~kJ} \mathrm{~mol}^{-1}$. For instance, by the same vdW-DF approach, the $E_{\mathrm{b}}$ of $\mathrm{H}_{2}$ is about $9.8-10.4 \mathrm{~kJ} \mathrm{~mol}^{-1}$ for $\mathrm{M}_{2}(\mathrm{BDC})_{2}$ (TED) series, where $\mathrm{M}$ is $\mathrm{Zn}, \mathrm{Mg}, \mathrm{Ni}^{45}$

To understand the interaction of the $\mathrm{H}_{2}$ molecule in the CoMIL-88A structure, we analyzed the charge density difference of the absorbed $\mathrm{H}_{2}$ molecule and the MOF, which is shown in Fig. 4. On the hollow, the ligand, and the metal side-on, the $\mathrm{H}_{2}$ molecule interacts with the MOF through its bonding state, while on the metal end-on site the interaction is via the antibonding state of $\mathrm{H}_{2}$. The charge exchange cloud of the $\mathrm{H}_{2}$ molecule closest to the MOF shows a charge gain for the cases of the $\mathrm{H}_{2}$ molecule adsorbed on the ligand, the metal side-on, and the metal end-on; however, it shows a charge donation for the most favorable adsorption site, i.e., the hollow site of the cobalt metal oxide. The size of the charge exchange cloud is qualitatively proportional to the binding strength of $\mathrm{H}_{2}$ with the MOF, see Table 3 for the binding energy. The hollow configuration (Fig. 4a) has the largest charge exchange cloud due to the strongest interaction with the MOF, while the metal end-on configuration (Fig. 4d) has the smallest charge exchange cloud because of the weakest interaction.

We also calculated the Bader charge exchange of the $\mathrm{H}_{2}$ molecule, see Table 3. We find that the Bader charge of the $\mathrm{H}_{2}$ molecule is very small and within the error of the charge calculation of $0.0005 \mathrm{e}$. Therefore, we can conclude that there is no significant charge transfer between the $\mathrm{H}_{2}$ molecule and the Co-MIL-88A due to the weak physisorption of the $\mathrm{H}_{2}$ molecule. This result is also in good agreement with the prediction from the small $\mathrm{H}_{2}$ stretching frequency shift obtained in the above part.

Deeper insights of the $\mathrm{MOF}-\mathrm{H}_{2}$ interaction can be exposed through the analysis of the electronic density of states. Fig. 5 shows that the $\mathrm{H}_{2}$ molecule interacts with the Co atoms of the Co-MIL-88A, where the state of $\mathrm{H}_{2}$ overlaps with the d orbital of the Co atoms. For the hollow, the ligand, and the metal side-on configurations, the $\mathrm{d}_{x y}, \mathrm{~d}_{x^{2}-y^{2}}$ and $\mathrm{d}_{z^{2}}$ orbitals of the metal atoms mainly contribute to the interaction with the hydrogen molecule, while it is the $\mathrm{d}_{z^{2}}$ orbital for the metal end-on configuration. The $s$ orbital of the metal atoms of the MOF also contributes to the interaction with $\mathrm{H}_{2}$ but most substantially for the most favorable $\mathrm{H}_{2}$ adsorption configuration, on the hollow site. Although the $\mathrm{H}_{2}$ molecule at the hollow site is far away from the nearest Co atoms with the average distance of $4.15 \AA$, the interaction of the $\mathrm{H}_{2}$ molecule and the Co atoms is still possible through the indirect interaction with the oxygen atoms in the outer space of the metal oxide. Fig. S1 in ESI $\dagger$ shows that

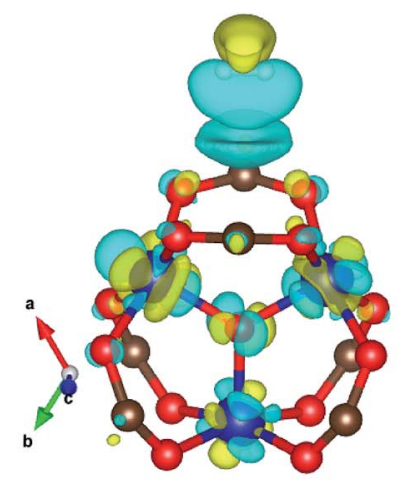

a) Hollow site

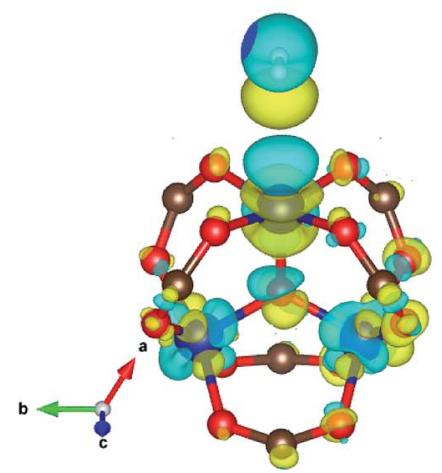

c) Metal (side-on) site

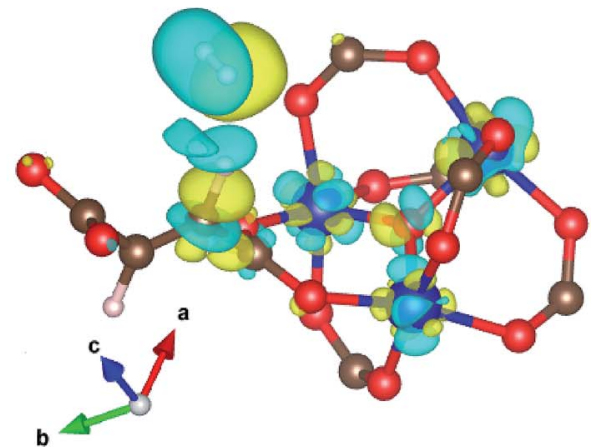

b) Ligand site

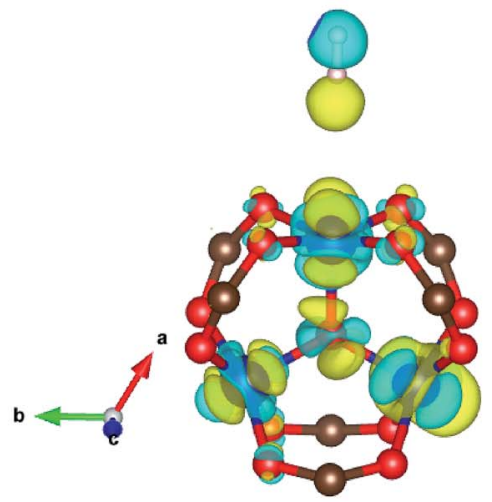

d) Metal (end-on) site

Fig. 4 The charge density difference for the favorable adsorption configuration of $\mathrm{H}_{2}$ in the Co-MIL-88A. Yellow and cyan clouds represent charge gain and loss, respectively. Isosurface $=2 \times 10^{-4}$ e bohr $^{-3}$. 

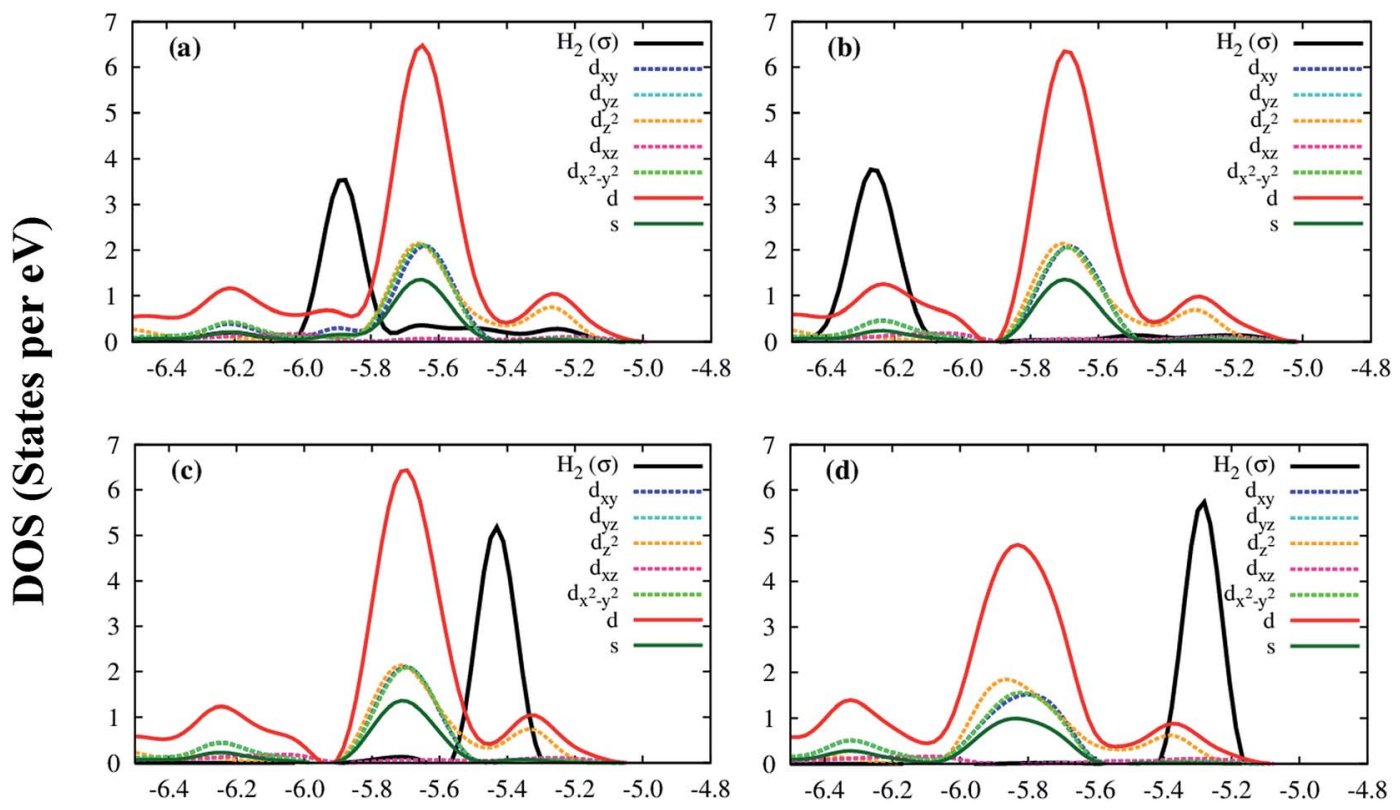

Fig. 5 The electronic density of state of the hydrogen molecule and the s and d orbitals of the Co atoms of the Co-MIL-88A at the sites: hollow (a), ligand (b), metal side-on (c), and metal end-on (d).

the charge cloud of the Co atoms mixes with the charge cloud of the oxygen atoms, which expands in a large area and approaches the charge cloud of the $\mathrm{H}_{2}$ molecule. Fig. 6 shows a more visible evidence of the interaction of the $\mathrm{H}_{2}$ molecule with the Co atoms through the real-space wave functions. ${ }^{46} \mathrm{We}$ can see that there is an overlapping between the wave function of the $\mathrm{H}$ atoms of the $\mathrm{H}_{2}$ molecule and that of the Co atoms. Furthermore, the $\mu_{3}-\mathrm{O}$ oxygen atoms of the Co-MIL-88A were also found to participate in the interaction with $\mathrm{H}_{2}$ via their $\mathrm{p}_{x}$ and $\mathrm{p}_{y}$ orbitals, see Fig. S2 in ESI. $\dagger$

Although the $\mathrm{Co}$ and $\mu_{3}-\mathrm{O}$ atoms can interact with the hydrogen molecule, the most profound interaction is from the $\mathrm{p}_{x}, \mathrm{p}_{y}, \mathrm{p}_{z}$ orbitals of all atoms of the Co-MIL-88A. Fig. 7 shows that a large portion of total $p$ DOS overlaps with the $\mathrm{H}_{2}$ DOS, which covers almost the whole area of the $\mathrm{H}_{2}$ DOS. This result implies that the p orbital of all atoms of the Co-MIL-88A should influence on the hydrogen adsorption more significantly compared to the s and d orbitals of the Co atoms.
Remarkably, we can quantitatively assess the interaction strength of $\mathrm{H}_{2}$ with the MOF by calculating a common area of DOS, ${ }^{47}$ which is the overlap area between the DOS of the adsorbed $\mathrm{H}_{2}$ and the DOS of the Co-MIL-88A as demonstrated in Fig. 8a. Here, we calculate the common area between the DOS of the adsorbed $\mathrm{H}_{2}$ molecule and the total DOS from all orbitals of the Co-MIL-88A. The results for the different adsorption sites can be seen in the seventh column of Table 5 and are plotted versus the binding energy in Fig. $8 \mathrm{~b}$. It shows that this quantity correlates to some extent with the binding energy, i.e., the larger the common area, the stronger the binding strength is. Furthermore, Fig. 5 and 7 also show that the more stable the $\mathrm{H}_{2}$ adsorption configuration becomes, the lower the peak height of the $\mathrm{H}_{2}$ DOS is. By calculating the DOS area of the adsorbed $\mathrm{H}_{2}$ molecule, listed in the last column of Table 5 and shown in Fig. $8 \mathrm{~b}$, we found that this peak area is inversely proportional to the common area of DOS.

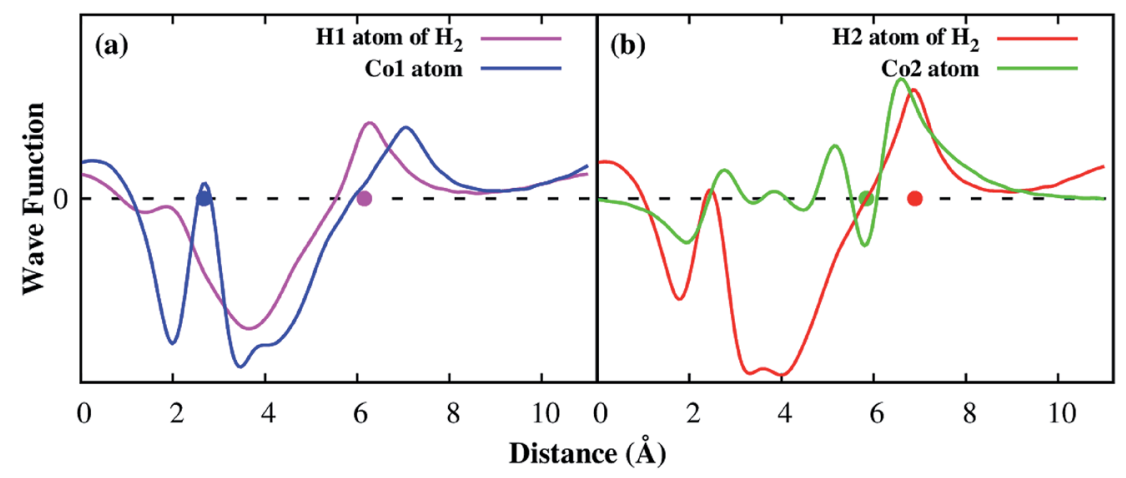

Fig. 6 The real part of the wave functions of the hydrogen atom and the Co atom along $x$ direction, the vector $a$ in Fig. 1a. The dot denotes for the position of the atoms along the $x$ direction. 

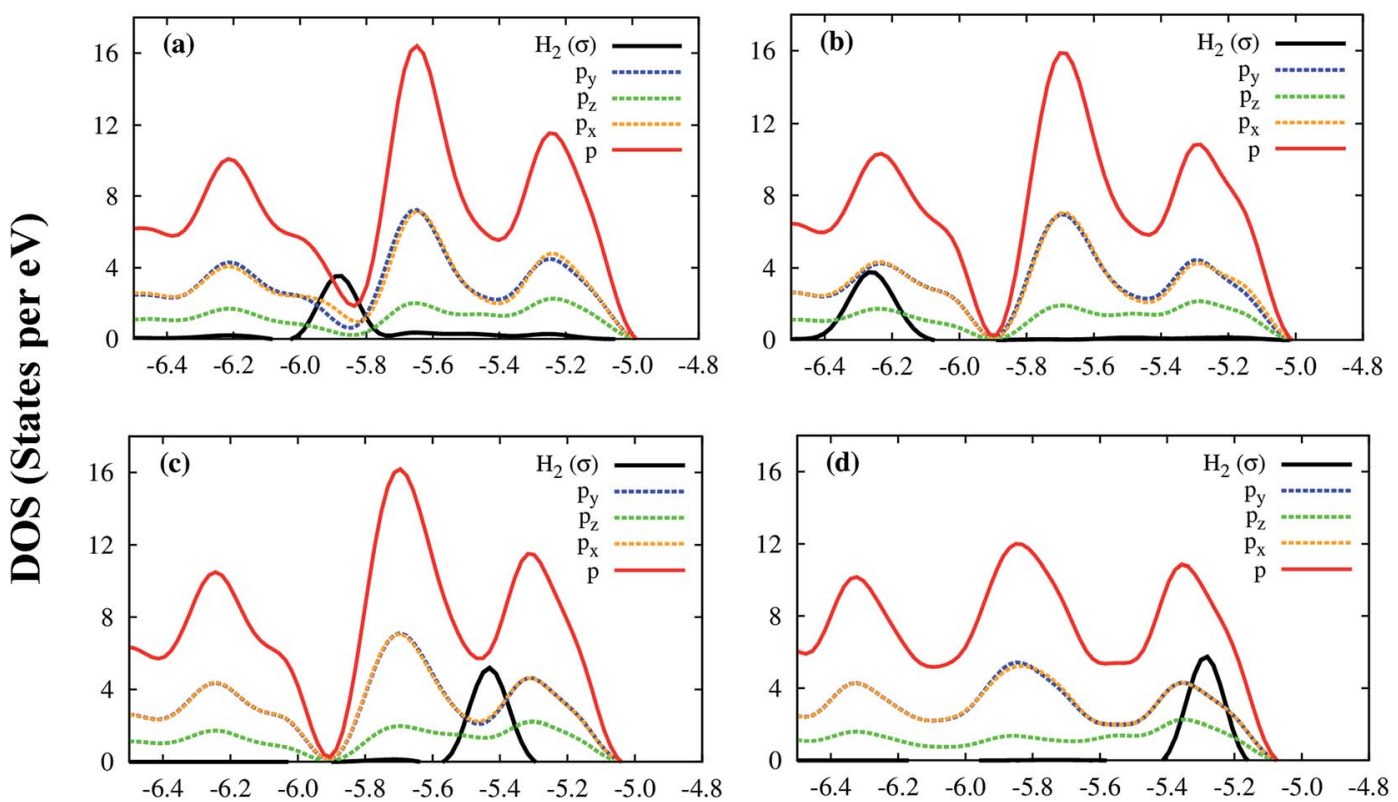

Fig. 7 The electronic density of state of the hydrogen molecule and the p orbitals of all atoms of Co-MIL-88A at the sites: hollow (a), ligand (b), metal side-on (c), and metal end-on (d).
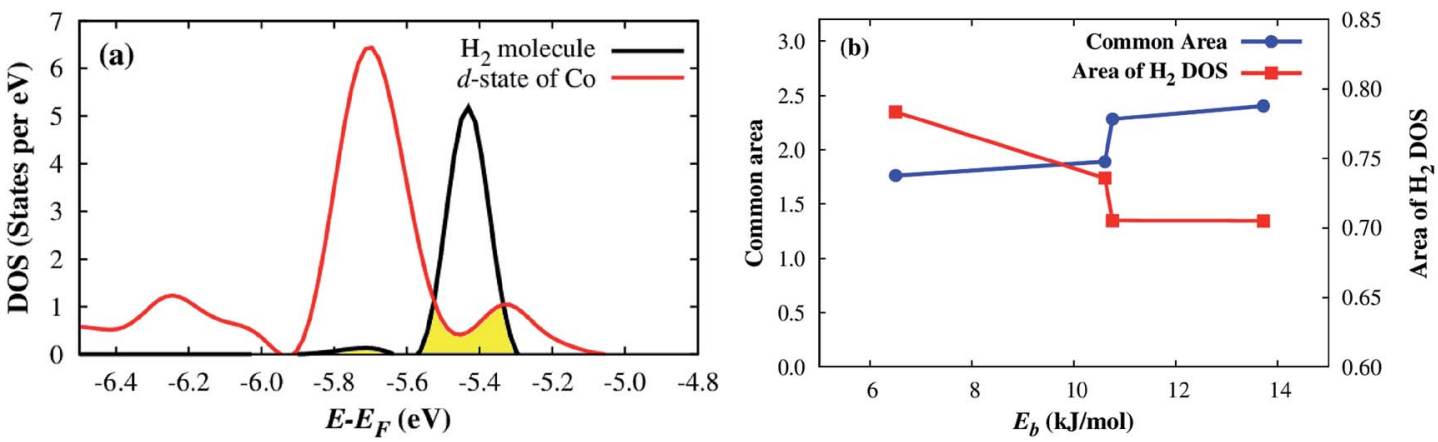

Fig. 8 A demonstration of the common area of DOS, the filled area (a), and the correlation between the common area of the $\mathrm{H}_{2} \mathrm{DOS}$ and the total DOS of all atoms of the Co-MIL-88A and the DOS area of the adsorbed $\mathrm{H}_{2}$ molecule with the binding energy (b).

We can break the contribution to the common area of the total DOS of all atoms into different portions that are from the $\mathrm{s}$ and d orbitals of Co atoms and from the total $\mathrm{p}$ orbital of all atoms of the MOF. We also calculated the common area of the $\mathrm{H}_{2}$ DOS and the total s orbital of oxygen and carbon atoms; however, there is no significant contribution to the interaction with the $\mathrm{H}_{2}$ molecule. Table 5 shows that although the contribution from the total p orbital of all atoms is large, the overlapping with the total $\mathrm{p}$ orbital of all atoms decreases while the overlapping with the $\mathrm{s}$ and $\mathrm{d}$ orbitals of Co atoms increase significantly for the more stable adsorption configurations. We also found that the common area of the $\mathrm{H}_{2}$ DOS with the $\mathrm{s}$ orbital and the $\mathrm{d}$ orbital of Co atoms and the p orbital of the $\mu_{3}-$ $\mathrm{O}$ atoms monotonically increase in the order: the metal end-on $<$ the metal side-on $<$ the ligand $<$ the hollow site. In generally speaking, the inner atoms of the trimers such as Co and $\mu_{3}-\mathrm{O}$ become more and more important for stabilizing the $\mathrm{H}_{2}$ adsorption. With the electronic analysis, we could explain the site dependence of the $\mathrm{H}_{2}$ adsorption.

\subsection{Hydrogen adsorption isotherms}

In the previous section, we provided some insight toward the $\mathrm{H}_{2}$ adsorption in the MOF. Here, we will quantitatively assess the storage capability of the Co-MIL-88A by calculating the hydrogen adsorption isotherms using GCMC simulations. The best way of performing GCMC simulations is to use the force field with both the dispersion and electrostatic interactions that were parameterized on the same footing from the DFT calculations. However, the parameterization of only the Coulomb interaction from the DFT while the parameters of the dispersion term was taken from the generic force field for $\mathrm{MOFs}^{33}$ which has also been used by several works in the literature. ${ }^{48-50}$ In this work, we assigned the point charges in an independent way from the dispersion force field parameters. Before applying the generic force field for $\mathrm{MOFs}^{33}$ and the DDEC charge assignment to the present problem, we first test the reliability of the force field through the calculation of the adsorption isotherm for a known MOF, the Co-MOF-74, and compare our simulated 
Table 5 Common area between the DOS of the adsorbed hydrogen molecule with the DOS of different components of the Co-MIL-88A

\begin{tabular}{|c|c|c|c|c|c|c|c|}
\hline Sites & $\begin{array}{l}\text { d orbital } \\
\text { of Co }\end{array}$ & $\begin{array}{l}\text { s orbital } \\
\text { of Co }\end{array}$ & $\begin{array}{l}\text { s and d orbitals } \\
\text { of Co }\end{array}$ & $\begin{array}{l}\text { p orbital of } \\
\mu_{3}-\mathrm{O} \text { atoms }\end{array}$ & $\begin{array}{l}\text { Total p orbital } \\
\text { of all atoms }\end{array}$ & $\begin{array}{l}\text { Total DOS of } \\
\text { all atoms }\end{array}$ & $\begin{array}{l}\text { Area of } \\
\mathrm{H}_{2} \text { DOS }\end{array}$ \\
\hline Hollow & 0.750 & 0.378 & 1.128 & 0.793 & 1.277 & 2.405 & 0.7050 \\
\hline Metal side-on & 0.376 & 0.081 & 0.457 & 0.250 & 1.433 & 1.890 & 0.7358 \\
\hline Metal end-on & 0.258 & 0.017 & 0.275 & 0.049 & 1.488 & 1.763 & 0.7836 \\
\hline
\end{tabular}

${ }^{a}$ We do not list the common area of the $\mathrm{H}_{2}$ DOS with the $\mathrm{O}$ p (except for $\mu_{3}-\mathrm{O}$ p) and $\mathrm{C}$ p orbitals separately because there are no simple rules for them.

result with the available experimental data. ${ }^{21}$ The hydrogen adsorption isotherm of the Co-MOF-74 framework was calculated at the temperature of $77 \mathrm{~K}$ and the pressure below 6 bar. Fig. 9a shows this result together with the experimental data. We found that the calculated excess $\mathrm{H}_{2}$ adsorption isotherms using the generic force field and the DDEC charge assignment are in good agreement with the experimental result at low pressures. Although there is a discrepancy at high pressures, the behavior of the isotherm is well reproduced by our scheme. Besides, to determine the reliability of the charge assignment while remaining the other force field parameters, we simulated the hydrogen adsorption isotherms for the Co-MOF-74 with the atomic point charges obtained by the DDEC charge calculation of our group and the charges (available for Co-MOF-74) taken from the library of RASPA, see Table S2. $\dagger$ Fig. $9 \mathrm{~b}$ shows that both results are in excellent agreement. Because the Co-MIL-88A and the Co-MOF-74 have the same characteristics such as having unsaturated metal sites, hexagonal unit cell, and containing the same types of elements, i.e., Co, C, O, and H. Therefore, we use the tested force field for the Co-MIL-88A.

For evaluating the adsorption capacity of $\mathrm{H}_{2}$ in the Co-MIL$88 \mathrm{~A}$, we computed the average amount of absolute and excess loadings at the temperatures of $77 \mathrm{~K}$ and $298 \mathrm{~K}$, and for pressures up to 100 bar. Fig. 10a shows that the $\mathrm{H}_{2}$ adsorption isotherms at $77 \mathrm{~K}$ increase sharply below 5 bar and achieves the maximum value of about $4.0 \mathrm{wt} \%$ at about 15 bar for the excess loading but still increases slightly for the absolute loading until 50 bar where the total uptake reaches the value of $4.5 \mathrm{wt} \%$. From these results, we see the $\mathrm{H}_{2}$ uptake in Co-MIL-88A at the cryogenic temperature $(77 \mathrm{~K})$ is moderate compared with that of the best MOFs reported up to now, see the recent review papers..$^{11,12}$ For example, MOF-5 (the MOF was firstly synthesized and was evaluated for hydrogen storage capacity in 2003) has the total uptake with $4.5 \mathrm{wt} \%$ at $(78 \mathrm{~K}, 1 \mathrm{bar})^{51}$ and $5.6 \mathrm{wt} \%$ at $(77 \mathrm{~K}, 100$ bar). ${ }^{49}$ At $77 \mathrm{~K}$, the highest excess $\mathrm{H}_{2}$ storage capacity is 9.95 $\mathrm{wt} \%$ at 56 bar and the absolute one is $16.4 \mathrm{wt} \%$ at 70 bar for NU$100 ;{ }^{52}$ while, the highest total $\mathrm{H}_{2}$ storage capacity is $17.6 \mathrm{wt} \%$ (excess $8.6 \mathrm{wt} \%$ ) at 80 bar for MOF-210..$^{53}$ Besides, several MOFs were also evaluated with a high $\mathrm{H}_{2}$ uptake of $7.5 \mathrm{wt} \%$ at $70 \mathrm{bar}$ for MOF-177, ${ }^{54} 2.4 \mathrm{wt} \%$ and $4.6 \mathrm{wt} \%$ at 31 bar for UiO-66 and UiO-67, respectively. ${ }^{55}$ The sudden increase of the adsorption isotherm at low pressures implies that the storage is mainly based on the adsorption of $\mathrm{H}_{2}$ with the MOF.

At room temperature, the isotherms increase fairly close to a linear function of the pressure, but are not saturated at our highest pressure of 100 bar (Fig. 10b). This result implies that the Co-MIL-88A is very stable and suitable for the hydrogen storage at high pressures. The maximum value for the excess and absolute loadings at $298 \mathrm{~K}$ and $100 \mathrm{bar}$ is 0.22 and 0.63 wt $\%$, respectively. The calculated absolute loading is comparable to the experimental data obtained for the best MOFs up to date at the standard condition of $298 \mathrm{~K}$ and 100 bar. For examples, the absolute loadings are $2.3 \mathrm{wt} \%$ for Be-BTB, ${ }^{56} 0.8$ $\mathrm{wt} \%$ for Mg-MOF- $74,{ }^{57}$ and the excess loadings are $0.62 \mathrm{wt} \%$ for MOF- $177,{ }^{54}$ at $298 \mathrm{~K}$ and $100 \mathrm{bar}$, many MOFs reported up to now also reached the $\mathrm{H}_{2}$ storage capacities of $0.36-0.58 \mathrm{wt} \% .^{58}$
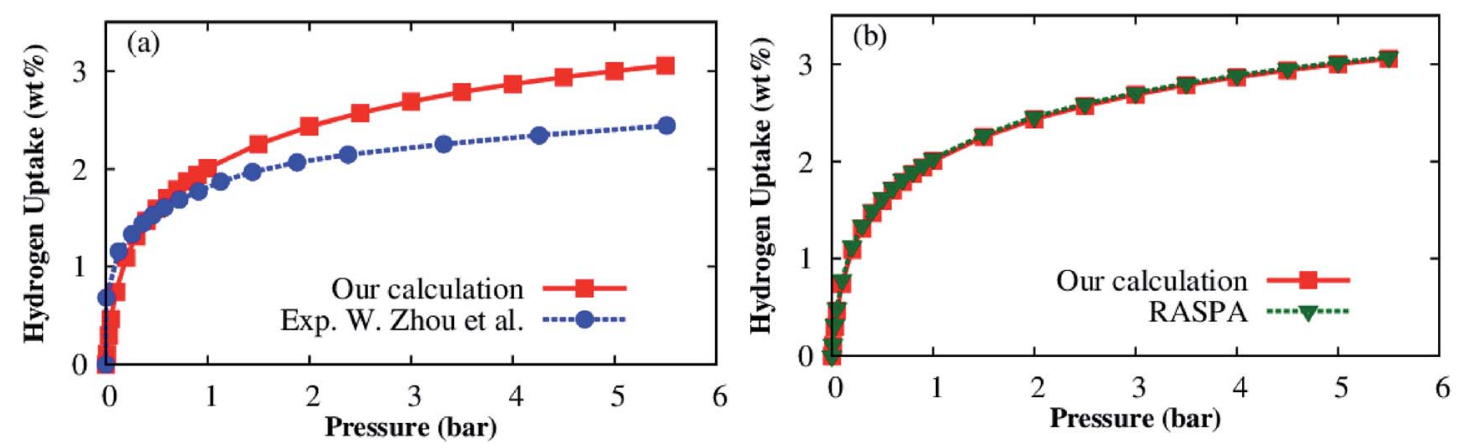

Fig. 9 Excess hydrogen uptake for Co-MOF-74 at $77 \mathrm{~K}$ : (a) GCMC simulation with the generic force field for MOFs and the DDEC charge assignment (solid square) and the experimental data (solid circle) extracted with permission from ref. 21. Copyright 2008 American Chemical Society; (b) GCMC simulation with the DDEC charges of this work and the charges taken from the library of RASPA. 

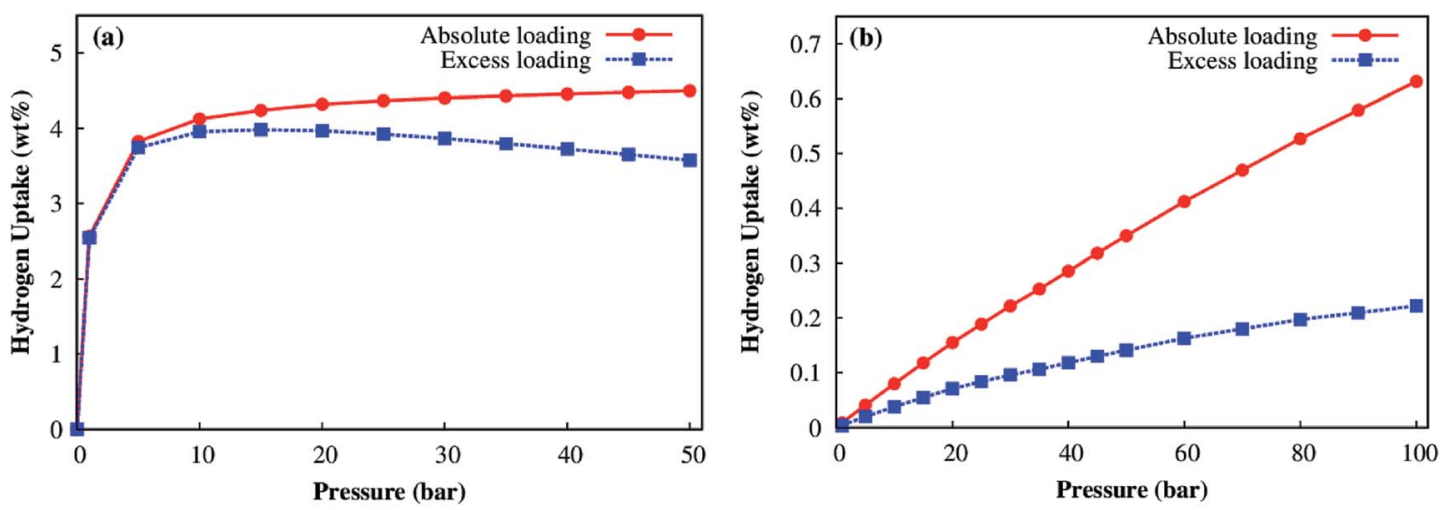

Fig. 10 Absolute (red) and excess (blue) adsorption isotherms for the Co-MIL-88A at $77 \mathrm{~K}$ (a) and $298 \mathrm{~K}$ (b).

Table 6 Absolute hydrogen uptake (wt\%) in the Co-MIL-88A with and without electrostatic interaction at $77 \mathrm{~K}$

\begin{tabular}{|c|c|c|c|c|}
\hline $\begin{array}{l}\text { Pressure } \\
\text { (bar) }\end{array}$ & $\begin{array}{l}\mathrm{LJ}+\text { Coulomb } \\
\text { interaction } \\
(\mathrm{wt} \%)\end{array}$ & $\begin{array}{l}\mathrm{LJ} \text { interaction } \\
\text { (wt\%) }\end{array}$ & $\begin{array}{l}\text { Coulomb } \\
\text { interaction } \\
(\mathrm{wt} \%)\end{array}$ & $\begin{array}{l}\text { Weight of } \\
\text { electrostatic } \\
\text { interaction (\%) }\end{array}$ \\
\hline 1 & 2.56 & 1.90 & 0.66 & 25.78 \\
\hline 2 & 3.21 & 2.53 & 0.68 & 21.18 \\
\hline 3 & 3.52 & 2.83 & 0.69 & 19.60 \\
\hline 4 & 3.69 & 3.01 & 0.68 & 18.43 \\
\hline 5 & 3.81 & 3.15 & 0.66 & 17.32 \\
\hline 10 & 4.13 & 3.52 & 0.61 & 14.77 \\
\hline 20 & 4.32 & 3.81 & 0.51 & 11.81 \\
\hline 30 & 4.40 & 3.97 & 0.43 & 9.77 \\
\hline 40 & 4.46 & 4.06 & 0.40 & 8.97 \\
\hline 50 & 4.50 & 4.14 & 0.36 & 8.00 \\
\hline
\end{tabular}

To assess the weight of the electrostatic interaction with respect to the dispersion one, we calculated the hydrogen uptake with and without the assignment of the atomic DDEC charges by our DFT calculations. The information was listed in Table 6, see also Fig. S3 and Tables S3 and S4.† Looking at the last two columns of Table S4, $\uparrow$ we found that the electrostatic interaction contributes less than $10 \%$ to the overall hydrogen uptakes at the room temperature. Table 6 shows that the contribution of the electrostatic interaction is much more significant at the low temperature than at the room temperature. Simultaneously, the electrostatic contribution decreases as the pressure increases. The dispersion interaction is more dominant than the electrostatic part.

Fig. S4 $\uparrow$ shows that the hydrogen molecules are more concentrated around the hollow than around the ligand and the metal site, which means that the hollow site is the most favorable adsorption place of the hydrogen gas. The average distance from the $\mathrm{H}_{2}$ molecules to the nearest atoms of the MOF, shown in Table S5 in ESI, $\uparrow$ was found to be in good agreement with that obtained by the density functional theory calculation, listed in Table 3. These results demonstrated that the used generic force field for MOFs could qualitatively reproduce the observation of the density functional theory results.

\section{Conclusions}

Our DFT-based results show that the favorable adsorption sites of $\mathrm{H}_{2}$ in Co-MIL-88A MOF is in the order: hollow > ligand > metal side-on $>$ metal end-on, in which the adsorption of $\mathrm{H}_{2}$ is most favorable on the hollow of four oxygen atoms with the binding energy of $13.72 \mathrm{~kJ} \mathrm{~mol}^{-1}$. This value is greater than that of many MOFs reported so far $\sim 4-13 \mathrm{~kJ} \mathrm{~mol}^{-1}$. The interaction between the bonding state of $\mathrm{H}_{2}$ with the $\mathrm{s}$ and d orbitals of the Co atoms and the $\mathrm{p}$ orbital of the $\mu_{3}-\mathrm{O}$ atoms favors the adsorption of the $\mathrm{H}_{2}$ molecule at the hollow site. Furthermore, the interaction of $\mathrm{H}_{2}$ with Co-MIL-88A is physisorption. The dispersion force dominates the interaction energy, which binds $\mathrm{H}_{2}$ at the adsorption sites.

At $77 \mathrm{~K}$, the excess adsorption isotherm of hydrogen gas in Co-MIL-88A saturates at 15 bar with $4.0 \mathrm{wt} \%$, but the absolute adsorption isotherm still increases slightly up to 50 bar and reach the maximum value of $4.5 \mathrm{wt} \%$. At the room temperature of $298 \mathrm{~K}$, the $\mathrm{H}_{2}$ adsorption isotherms increase strongly upon the increase of the temperature and reach 0.63 and $0.22 \mathrm{wt} \%$ for the absolute and excess uptakes at 100 bar, respectively. These achieved results are worthy for hydrogen storage. Further study will be on the enhancement of the $\mathrm{H}_{2}$ storage capacity by substituting metal or linker of MIL-88A.

\section{Conflicts of interest}

There are no conflicts of interest to declare.

\section{Acknowledgements}

This research was funded by Ho Chi Minh City University of Technology - VNU-HCM under grant number TNCS-2015KHUD-33. One of the authors (Do Ngoc Son) acknowledges the financial support of the Vietnam National Foundation for Science and Technology Development (NAFOSTED) under grant number 103.01-2017.04. We acknowledge the usage of the computer time and software granted by the Institute of Physical Chemistry of Romanian Academy, Bucharest (HPC infrastructure developed under the projects Capacities $84 \mathrm{Cp} / \mathrm{I}$ of 15.09.2007 and INFRANANOCHEM 19/01.03.2009). 


\section{References}

1 Z. Chang, D.-S. Zhang, Q. Chen and X.-H. Bu, Phys. Chem. Chem. Phys., 2013, 15, 5430-5442.

2 S. Ma and H.-C. Zhou, Chem. Commun., 2010, 46, 44-53.

3 M. Bastos-Neto, C. Patzschke, M. Lange, J. Möllmer, A. Möller, S. Fichtner, C. Schrage, D. Lässig, J. Lincke, R. Staudt, H. Krautscheid and R. Gläser, Energy Environ. Sci., 2012, 5, 8294-8303.

4 B. Panella and M. Hirscher, in Handbook of Hydrogen storage: New materials for future energy, ed. M. Hirscher, Wiley-VCH Verlag GmbH \& Co. KGaA, Weinheim, 2010, pp. 39-62.

5 K. Mazloomi and C. Gomes, Renewable Sustainable Energy Rev., 2012, 16, 3024-3033.

6 J. Goldsmith, A. G. Wong-Foy, M. J. Cafarella and D. J. Siegel, Chem. Mater., 2013, 25, 3373-3382.

7 D. A. Gomez and G. Sastre, Phys. Chem. Chem. Phys., 2011, 13, 16558-16568.

8 D. Zhao, D. Yuan and H. Zhou, Energy Environ. Sci., 2008, 1, 222-235.

9 J. Sculley, D. Yuan and H. Zhou, Energy Environ. Sci., 2011, 4, 2721-2735.

10 H. W. Langmi, J. Ren, B. North, M. Mathe and D. Bessarabov, Electrochim. Acta, 2014, 128, 368-392.

11 M. P. Suh, H. J. Park, T. K. Prasad and D.-W. Lim, Chem. Rev., 2012, 112, 782-835.

12 T. T. T. Huong, P. N. Thanh, N. T. X. Huynh and D. N. Son, VNU Journal of Science: Mathematics-Physics, 2016, 32, 67-85.

13 L. J. Murray, M. Dinca and J. R. Long, Chem. Soc. Rev., 2009, 38, 1294-1314.

14 D. Sahu, P. Mishra, S. Edubilli, A. Verma and S. Gumma, J. Chem. Eng. Data, 2013, 58, 3096-3101.

15 P. Horcajada, F. Salles, S. Wuttke, T. Devic, D. Heurtaux, A. Vimont, M. Daturi, O. David, E. Magnier, N. Stock, Y. Filinchuk, D. Y. Popov, C. Riekel, G. Férey, C. Serre, G. Maurin and D. Popov, J. Am. Chem. Soc., 2011, 133, 17839-17847.

16 S. S. Han, J. L. Mendoza-Cortes and W. A. Goddard, Chem. Soc. Rev., 2009, 38, 1460-1476.

17 J. G. Vitillo, L. Regli, S. Chavan, G. Ricchiardi, G. Spoto, P. D. C. Dietzel, S. Bordiga and A. Zecchina, J. Am. Chem. Soc., 2008, 130, 8386-8396.

18 A. C. McKinlay, J. F. Eubank, S. Wuttke, P. S. Wheatley, P. Bazin, J.-C. Lavalley, M. Daturi, A. Vimont, G. De Weireld, P. Horcajada, C. Serre and R. E. Morris, Chem. Mater., 2013, 25, 1592-1599.

19 S. Wongsakulphasatch, W. Kiatkittipong, J. Saupsor, J. Chaiwiseshphol, P. Piroonlerkgul, V. Parasuk and S. Assabumrungrat, Greenhouse Gases: Sci. Technol., 2016, 12, 1-12.

20 D. Gygi, E. D. Bloch, J. A. Mason, M. R. Hudson, M. I. Gonzalez, R. L. Siegelman, T. A. Darwish, W. L. Queen, C. M. Brown and J. R. Long, Chem. Mater., 2016, 28, 1128-1138.

21 W. Zhou, H. Wu and T. Yildirim, J. Am. Chem. Soc., 2008, 130, 15268-15269.
22 J. W. Yoon, Y. Seo, Y. K. Hwang, J. Chang, H. Leclerc, S. Wuttke, P. Bazin, A. Vimont, M. Daturi, E. Bloch, P. L. Llewellyn, C. Serre, P. Horcajada, J. Greneche, A. E. Rodrigues and G. Ferey, Angew. Chem., Int. Ed., 2010, 49, 5949-5952.

23 G. Kresse and J. Furthmüller, Phys. Rev. B, 1996, 54, 1116911186.

24 J. P. Perdew, K. Burke and M. Ernzerhof, Phys. Rev. Lett., 1996, 77, 3865-3868.

25 J. P. Perdew, K. Burke and M. Ernzerhof, Phys. Rev. Lett., 1997, 78, 1396.

26 P. E. Blochl, Phys. Rev. B, 1994, 50, 17953-17979.

27 G. Kresse and D. Joubert, Phys. Rev. B, 1999, 59, 1758-1775.

28 J. D. Pack and H. J. Monkhorst, Phys. Rev. B, 1976, 13, 51885192.

29 M. Methfessel and A. T. Paxton, Phys. Rev. B, 1989, 40, 36163621.

30 P. E. Blochl, O. Jepsen and O. K. Andersen, Phys. Rev. B, 1994, 49, 16223-16233.

31 T. A. Manz and D. S. Sholl, J. Chem. Theory Comput., 2012, 8, 2844-2867.

32 W. Tang, E. Sanville and G. Henkelman, J. Phys.: Condens. Matter, 2009, 21, 84204.

33 D. Dubbeldam, S. Calero, D. E. Ellis and R. Q. Snurr, Mol. Simul., 2016, 42, 81-101.

34 M. Dion, H. Rydberg, E. Schröder, D. C. Langreth and B. I. Lundqvist, Phys. Rev. Lett., 2004, 92, 246401.

35 F. D. Murnaghan, Finite deformation of an elastic solid, Wiley, New York, 1951.

36 S. Surble, C. Serre, C. Mellot-Draznieks, F. Millange and G. Ferey, Chem. Commun., 2006, 284-286.

37 T. Sagara, J. Klassen and E. Ganz, J. Chem. Phys., 2004, 121, 12543-12547.

38 T. Mueller and G. Ceder, J. Phys. Chem. B, 2005, 109, 1797417983.

39 D. a. Gomez, A. F. Combariza and G. Sastre, Phys. Chem. Chem. Phys., 2009, 11, 9250-9258.

40 T. B. Lee, D. Kim, D. H. Jung, S. B. Choi, J. H. Yoon, J. Kim, K. Choi and S. H. Choi, Catal. Today, 2007, 120, 330-335.

41 S. S. Han, W. Q. Deng and W. A. Goddard, Angew. Chem., Int. Ed., 2007, 46, 6289-6292.

42 X. Solans-Monfort, M. Sodupe, C. M. Zicovich-wilson, E. Gribov, G. Spoto, C. Busco and P. Ugliengo, J. Phys. Chem. B, 2004, 108, 8278-8286.

43 S. Satyapal, J. Petrovic, C. Read, G. Thomas and G. Ordaz, Catal. Today, 2007, 120, 246-256.

44 D. A. Gomez, J. Toda and G. Sastre, Phys. Chem. Chem. Phys., 2014, 16, 19001-19010.

45 N. Nijem, J.-F. Veyan, L. Kong, K. Li, S. Pramanik, Y. Zhao, J. Li, D. Langreth and Y. J. Chabal, J. Am. Chem. Soc., 2010, 132, 1654-1664.

46 R. M. Feenstra, N. Srivastava, Q. Gao, M. Widom, B. Diaconescu, T. Ohta, G. L. Kellogg, J. T. Robinson and I. V. Vlassiouk, Phys. Rev. B, 2013, 87, 041406(R).

47 R. Hoffmann, Rev. Mod. Phys., 1988, 60, 601-628.

48 C. E. Wilmer, R. Q. Snurr and M. Carlo, Chem. Eng. J., 2011, 171, 775-781. 
49 Q. Yang and C. Zhong, J. Phys. Chem. B, 2006, 110, 655-658. 50 D. Fairen-Jimenez, Y. J. Colón, O. K. Farha, Y.-S. Bae, J. T. Hupp and R. Q. Snurr, Chem. Commun., 2012, 48, 10496. 51 N. L. Rosi, J. Eckert, M. Eddaoudi, D. T. Vodak, J. Kim, M. O'Keeffe and O. M. Yaghi, Science, 2003, 300, 1127-1129.

52 O. K. Farha, A. Ö. Yazaydın, I. Eryazici, C. D. Malliakas, B. G. Hauser, M. G. Kanatzidis, S. T. Nguyen, R. Q. Snurr and J. T. Hupp, Nat. Chem., 2010, 2, 944-948.

53 H. Furukawa, N. Ko, Y. B. Go, N. Aratani, S. B. Choi, E. Choi, A. O. Yazaydin, R. Q. Snurr, M. O'Keeffe, J. Kim and O. M. Yaghi, Science, 2010, 329, 424-428.
54 Y. Li and R. T. Yang, Langmuir, 2007, 23, 12937-12944.

55 S. Chavan, J. G. Vitillo, D. Gianolio, O. Zavorotynska, B. Civalleri, S. Jakobsen, M. H. Nilsen, L. Valenzano, C. Lamberti, K. P. Lillerud and S. Bordiga, Phys. Chem. Chem. Phys., 2012, 14, 1614-1626.

56 W. X. Lim, A. W. Thornton, A. J. Hill, B. J. Cox, J. M. Hill and M. R. Hill, Langmuir, 2013, 29, 8524-8533.

57 K. Sumida, C. M. Brown, Z. R. Herm, S. Chavan, S. Bordiga and J. R. Long, Chem. Commun., 2011, 47, 1157-1159.

58 S. J. Yang, J. H. Im, H. Nishihara, H. Jung, K. Lee, T. Kyotani and C. R. Park, J. Phys. Chem. C, 2012, 116, 10529-10540. 